\title{
Perfil lipídico, antropométrico y condición física de estudiantes deportistas universitarios
}

\author{
Lipid anthropometric and physical condition profile of university athlete students
}

\author{
Diana María García-Cardona1* orcid.org/0000-0001-6026-9093 \\ Oscar Eduardo Sánchez-Muñoz'1 orcid.org/0000-0003-2438-3360 \\ Carlos Enrique Cabrera-Arismendy' orcid.org/0000-0001-5581-0543 \\ Beatriz Restrepo-Cortés ${ }^{2}$ orcid.org/0000-0001-9779-1870
}

1 Programa de Educación Física y Deportes, Universidad del Quindío. Armenia, Colombia

2 Programa de Medicina. Universidad del Quindío. Armenia, Colombia

Fecha de recepción: Noviembre 2-2016 Fecha de revisión: Marzo 24-2016 Fecha de aceptación: Agosto 11 - 2017

García-Cardona DM, Sánchez-Muñoz OE, Cabrera-Arismendy CE, Restrepo-Cortés B. Perfil lipídico, antropométrico y condición física de estudiantes deportistas universitarios. Univ. Salud. 2017;19(2):267-279. DOI: http://dx.doi.org/10.22267/rus.171902.89

\section{Resumen}

Introducción: Reconocer aspectos como perfil lipídico, composición corporal y composición física de los deportistas, puede evitar errores recurrentes en el entrenamiento deportivo. Objetivo: Establecer el perfil lipídico, antropométrico y de condición física de estudiantes pertenecientes a las selecciones deportivas de la Universidad del Quindío. Materiales y métodos: Se valoró a integrantes de 14 selecciones deportivas en sus medidas antropométricas acorde a los lineamientos de la International Society for the Avancement in Kineanthropometric (ISAK); para determinar fuerza, velocidad, resistencia y flexibilidad se aplicó diferentes test. Para establecer el perfil lipídico se utilizó kits comerciales. Resultados: La población presenta rangos normales de perfil lipídico. Respecto al perfil antropométrico, se encontró promedios de porcentaje graso sobre los considerados normales para deportistas, aunque corresponden a la normalidad de la población en general. A nivel condicional la flexibilidad fue la capacidad con promedios inferiores de los rangos considerados aceptables. Conclusión: Es necesario buscar estrategias que permitan mejorar aspectos como: niveles de porcentaje graso y flexibilidad, que en algunos participantes no están acorde con los rangos establecidos para deportistas de rendimiento.

Palabras clave: Lípidos; antropometría; atletas; educación superior. (Fuente: DeCS, Bireme).

\begin{abstract}
Introduction: Recognizing aspects such as lipid profile, body composition and physical composition of athletes can avoid recurrent errors in sports training. Objective: To establish the lipid, anthropometric and physical condition profile of students belonging to the sports teams of the University of Quindío. Materials and methods: Members of 14 sports teams were valued in their anthropometric measurements according to the guidelines of the International Society for the Advancement in Kinanthropometric (ISAK). For this, different tests were applied to determine strength, speed, resistance and flexibility and commercial kits were used to establish the lipid profile. Results: The population has normal ranges of lipid profile. Regarding the anthropometric profile, averages of fat percentage were found above those that are considered normal for athletes, although they correspond to the normality of the population in general. On a conditional level, flexibility was the capacity with lower averages from those considered as acceptable ranks. Conclusions: It is necessary to look for strategies to improve aspects such as: levels of fatty percentage and flexibility, which in some participants are not in line with the established ranges for performance athletes.
\end{abstract}


Keywords: lipids; anthropometry; athletes; higher education. (Source: DeCS, Bireme).

\section{Introducción}

Las características antropométricas son parte del conjunto de variables biológicas relacionadas con el rendimiento deportivo(1,2). Desde hace décadas, se conoce que el perfil antropométrico es un factor de selección muy importante. Por ello, la cineantropometría (medición de la composición corporal humana en función de los cambios en los estilos de vida, la nutrición y los niveles de actividad) aporta gran cantidad de información sobre la estructura del individuo en determinado momento y otorga la posibilidad de cuantificar las modificaciones causadas por el entrenamiento ${ }^{(3)}$.

Cada deporte, especialidad o modalidad, tiene un patrón cineantropométrico específico muy bien definido, en algunos casos por diversas investigaciones(4). Gracias a este patrón es posible conocer las características antropométricas que debería tener un determinado sujeto para alcanzar el éxito deportivo. Un deportista está supeditado a niveles de esfuerzo que lo obligan a superarse tanto individual como colectivamente desde el punto de vista, físico, técnico, táctico y psicológico para cumplir con las necesidades de la disciplina deportiva que ejecuta. Así, el reconocimiento de la condición física a través de evaluaciones (test deportivos) en especial, las capacidades condicionales (resistencia, fuerza, velocidad, flexibilidad), se hacen necesarias para evidenciar la forma deportiva que deben tener todos aquellos estudiantes universitarios que se preocupan por representar a su institución, sin embargo, para ello requieren de unas buenas cargas de trabajo que le permitirán competir de forma adecuada, desde el punto de vista energético, y a su vez, representar capacidad de dominio de las técnicas.

En la actualidad es ampliamente aceptado que el ejercicio físico mejora las condiciones de salud cardiovascular, esto se debe a que el ejercicio regula una serie de procesos fisiológicos a través de diversos mecanismos $(5,6)$, entre los cuales se encuentran la modulación de los lípidos plasmáticos y principalmente la elevación del colesterol HDL ${ }^{(7,8)}$. Sin embargo, en diversos estudios con personas jóvenes se ha observado la presencia de niveles alterados del perfil lipídico, al parecer asociados con la dieta. El estudio de Bogalusa $^{(9)}$ reporta que el peso, la adiposidad y la distribución de grasa no sólo se relacionan con niveles séricos alterados de lípidos y lipoproteínas sino que también contribuyen en una presentación temprana de resistencia a la insulina.

El no conocer aspectos tales como el perfil lipídico, la composición corporal y la condición física de los deportistas conduce a errores recurrentes en el desarrollo del entrenamiento deportivo, ya que en muchas ocasiones los deportistas pueden estar siendo sometidos a cargas de trabajo inapropiadas que pueden conllevar a desarrollar posibles problemas de salud. Por lo anterior, el presente estudio planteó como objetivo principal determinar el perfil lipídico, antropométrico y la condición física de deportistas de una universidad pública del departamento del Quindío.

\section{Materiales y métodos}

Se realizó un estudio descriptivo exploratorio de corte transversal. La población la constituyeron estudiantes que pertenecían a las selecciones deportivas (162 deportistas) de la Universidad del Quindío-Armenia Colombia. El estudio se hizo en hombres y mujeres voluntarios, mayores de 18 años que firmaron el consentimiento informado. Se excluyeron del estudio deportistas con enfermedad comprobada a través de su historia clínica. También se excluyeron los deportistas lesionados.

El total de participantes fue de 162 sujetos, dentro de los cuales habían 67 mujeres y 95 hombres, estos estuvieron distribuidos por selección de la siguiente manera: voleibol 10 hombres y 10 mujeres, fútbol 16 hombres y fútbol femenino 22 mujeres, baloncesto 9 hombres y 7 mujeres, porrismo 12 hombres y 6 mujeres, squash 7 hombres y 4 mujeres, tenis de mesa 6 hombres y 
4 mujeres, taekwondo 3 hombres y 3 mujeres, natación 10 hombres y 3 mujeres, microfútbol 8 mujeres, halterofilia 3 hombres, atletismo 5 hombres, triatlón 3 hombres, karate 6 hombres y tenis de campo 5 hombres. Para un total de 14 selecciones, con una frecuencia de entrenamiento de 4 días a la semana y una duración de dos horas cada sesión.

\section{Variables}

Como variables bioquímicas se tuvo el perfil lipídico (colesterol total (CT), triglicéridos (TG) y colesterol unido a las lipoproteínas de alta densidad (HDL), los dos primeros fueron cuantificados por métodos enzimáticos colorimétricos (Human®) y el último se valoró mediante separación selectiva inicial con ácido fosfotungstico/cloruro de magnesio (Human ${ }^{\circledR}$ )). La muestra sanguínea fue recolectada después de doce horas de ayuno, por punción venosa en dos tubos secos por personal especializado para tal fin, el suero se consiguió por centrifugación a $1000 \mathrm{~g}$ por 15 minutos, a $4^{\circ} \mathrm{C}$, separado en microtubos de $1,5 \mathrm{~mL}$ tipo eppendorf $\mathrm{y}$ almacenado a $-20^{\circ} \mathrm{C}$ hasta su uso.

Las variables antropométricas fueron, edad cronológica, edad morfológica, masa, talla, índice de masa corporal (IMC), índice cintura cadera (ICC), porcentaje residual, porcentaje óseo, porcentaje adiposo, porcentaje muscular y piel, para la valoración de las mismas se siguieron las indicaciones de la International Society for the Avancement in Kineanthropometric (ISAK) descritas por Marfell-Jones, Olds, Stewart y Carter(10). Antes de realizar las valoraciones se procedió al marcaje de los puntos anatómicos de referencia necesarios para la obtención de las medidas a estudiar, utilizando un lápiz demográfico. Los puntos anatómicos marcados se encuentran en las siguientes posiciones: acromial, radial, estiloideo, iliocrestal, ilioespinal, trocantéreo, tibial, ángulo infraescapular, abdominal lateral. En todos los casos las marcaciones fueron realizadas al lado derecho del sujeto. Las mediciones se tomaron partiendo de la posición antropométrica de referencia: masa, talla, talla sentada, pliegues cutáneos (tricipital, subescapular, supraespinal, abdominal, muslo y pierna), perímetros (brazo relajado, antebrazo, muslo 1 máximo, pierna, torácico, cefálico y cintura); diámetros (biacromial, transverso del tórax, antero-posterior del tórax, biliocrestal, bicondíleo de fémur y biepicondileo de húmero). Como variables de condición física se tuvieron en cuenta la velocidad, resistencia, flexibilidad y fuerza, que fueron valoradas con los test de aceleración de 30 metros, Legger, flexión anterior del tronco y el salto horizontal respectivamente(15).

\section{Análisis estadístico}

Se hizo un análisis descriptivo de cada una de las variables, los resultados se presentan con el promedio \pm la desviación estándar y los límites máximos y mínimos. Se hizo una comparación de cada una de las selecciones por sexo, para identificar las variables que tenían diferencia significativa, con un $\mathrm{P}$ valor $\leq 0,05$, utilizando como estadístico de prueba la T-Student. El análisis de los datos obtenidos se realizó con el software licenciado Statgraphics Centurion.

\section{Consideraciones éticas}

Para el desarrollo de esta investigación se tuvo en cuenta la Declaración de Helsinki y la Resolución 8430 del 4 de octubre de 1993, en la cual se establecen las normas científicas, técnicas y administrativas para la investigación en salud en Colombia(11). El estudio contó con la aprobación del Comité de Ética y Bioética de la Universidad del Quindío. Previo a la recolección de la información los sujetos de estudio diligenciaron el consentimiento informado.

\section{Resultados}

En la Tabla 1 se muestran las variables del perfil lipídico por sexo y por selección deportiva de las 14 selecciones caracterizadas. Se puede observar que en todas las selecciones y en ambos sexos se presenta en promedio un colesterol total (CT) por debajo de $200 \mathrm{mg} / \mathrm{dl}$, o sea dentro de los límites considerados como deseables ${ }^{(12)}$, sin embargo, se encontraron 2 hombres y una mujer que estaban por encima de este límite. Al hacer las comparaciones por sexo únicamente se encontró diferencia estadísticamente significativa por sexo en la selección de fútbol. Con respecto a los valores promedio de las lipoproteínas de baja 
densidad (LDL), en los deportistas de ambos sexos se observa que es menor de $100 \mathrm{mg} / \mathrm{dl}$, considerándolo un nivel óptimo; en los límites superiores de algunas de las selecciones se encuentran personas en un nivel cercano al óptimo (100-129 mg/dl) y límite alto (130-159 $\mathrm{mg} / \mathrm{dl})$; solo una mujer de la selección de baloncesto está en el rango de alto (160$189 \mathrm{mg} / \mathrm{dl})$ con un valor de LDL de $168 \mathrm{mg} / \mathrm{dl}(12)$.

Tabla 1. Variables de perfil lipídico por selección y sexo de los sujetos de estudio, Armenia, 2016

\begin{tabular}{|c|c|c|c|c|c|}
\hline \multirow[t]{3}{*}{ Selección } & \multicolumn{5}{|c|}{ Variables } \\
\hline & CT (mg/dl) & HDL (mg/dl) & LDL (mg/dl) & TG (mg/dl) & IA \\
\hline & F $\quad$ M & $\mathbf{F} \quad \mathbf{M}$ & $\mathbf{F} \quad \mathbf{M}$ & $\mathbf{F} \quad \mathbf{M}$ & $\mathbf{M}$ \\
\hline Voleibol & $\begin{array}{cr}149,8 \pm 19,4 & 155,7 \pm 29,9 \\
(123,6-189,9) & (128-203,2) \\
& P=0,9684\end{array}$ & $\begin{array}{rr}45,37 \pm 6,6 & 38,19 \pm 7,7 \\
(32,8-57) & (28,4-52,4) \\
& P=0,0648\end{array}$ & $\begin{array}{cr}88,66 \pm 12 & 5,58 \pm 23,1 \\
(71,5-114) & (62,7-128) \\
& P=0,9277\end{array}$ & $\begin{array}{rr}79 \pm 33 & 109,7 \pm 29 \\
(41,1-149) & (78,3-168,8) \\
& P=0,1158\end{array}$ & $\begin{array}{lr}3,3 \pm 0,3 & 4,15 \pm 0,8 \\
(2,8-3,7) & (3,3-5,6) \\
& P=0,0750\end{array}$ \\
\hline Futbol & $\begin{array}{rr}165,5 \pm 26,7 & 143,9 \pm 24,6 \\
(122,2-120,6) & (112,6-201,6) \\
& P=0,0269 \\
\end{array}$ & $\begin{array}{cr}46 \pm 7,8 & 39,6 \pm 8,1 \\
(34,6-60,6) & (24,3-53,1) \\
& P=0,0064\end{array}$ & $\begin{array}{rr}100,8 \pm 24 & 85,7 \pm 19 \\
(64,5-162) & (62,2-129) \\
& P=0,0795 \\
\end{array}$ & $\begin{array}{rr}94 \pm 8,4 & 93 \pm 8,3 \\
(51,1-223) & (39,4-213) \\
& P=0,8922\end{array}$ & $\begin{array}{cr}3,6 \pm 0,8 & 3,7 \pm 0,9 \\
(2,2-5,3) & (2,7-5,8) \\
& P=0,4916\end{array}$ \\
\hline Baloncesto & $\begin{array}{rr}151 \pm 42,7 & 148,8 \pm 24 \\
(105,7-233) & (120,6-186,6) \\
& P=0,8193\end{array}$ & $\begin{array}{lr}44,31 \pm 7,5 & 42,9 \pm 7,3 \\
(32,7-56) & (31,1-55) \\
& P=0,4549\end{array}$ & $\begin{array}{cr}93,6 \pm 3,0 & 89,7 \pm 20 \\
(63-168) & (68,4-124) \\
& P=0,7855\end{array}$ & $\begin{array}{cr}66,7 \pm 16 & 80,8 \pm 20,5 \\
(40,4-90,6) & (54-109,4) \\
& P=0,1375\end{array}$ & $\begin{array}{cr}3,4 \pm 0,8 & 3,6 \pm 0,9 \\
(2,3-4,6) & (2,9-5,7) \\
& P=0,6400\end{array}$ \\
\hline Porrismo & $\begin{array}{rr}132,2 \pm 25 & 166,2 \pm 15 \\
(105,6-190,7) & (142,2-189,4) \\
& P=0,1750 \\
\end{array}$ & $\begin{array}{rr}41,5 \pm 5,7 & 30,5 \pm 5,3 \\
(34,4-48,6) & (23,8-39,1) \\
& P=0,0101\end{array}$ & $\begin{array}{lr}82,1 \pm 21 & 104,4 \pm 17 \\
(53,4-100) & (86,3-136) \\
& P=0,2165\end{array}$ & $\begin{array}{cc}73 \pm 22 & 157,1 \pm 48 \\
(37,3-98,4) & (105-206) \\
& P=0,0163 \\
\end{array}$ & $\begin{array}{rr}3,3 \pm 0,5 & 4,5 \pm 0,8 \\
(2,5-4,1) & (3,4-6,4) \\
& P=0,0093\end{array}$ \\
\hline Squash & $\begin{array}{cr}171 \pm 30 & 141,3 \pm 40 \\
(148,2-213,1) & (88,5-173,3) \\
& P=0,3137 \\
\end{array}$ & $\begin{array}{lr}55,5 \pm 11,9 & 51,4 \pm 9,9 \\
(39,8-67,1) & (37,2-58) \\
& P=0,1758\end{array}$ & $\begin{array}{lr}99,3 \pm 20 & 78,4 \pm 33,8 \\
(79,3-126) & (39,8-110) \\
& P=0,5072 \\
\end{array}$ & $\begin{array}{rr}82 \pm 30 & 57,6 \pm 8,7 \\
(47,1-22) & (49,2-69,7) \\
& P=0,9997\end{array}$ & $\begin{array}{r}2,7 \pm 0,5 \\
(2,2-3,3) \\
P=0,6883\end{array}$ \\
\hline Tenis de mesa & $\begin{array}{cr}175,6 \pm 16,7 & 165,8 \pm 30 \\
(155,7-196,1) & (116,9-207,2) \\
& P=0,3283\end{array}$ & $\begin{array}{cr}56,01 \pm 9,1 & 40,6 \pm 3,3 \\
(40,6-61) & (36,8-43,7) \\
& P=0,0103\end{array}$ & $\begin{array}{lr}101 \pm 16,5 & 103,5 \pm 33 \\
(78,4-117) & (55,3-139) \\
& P=0,8731\end{array}$ & $\begin{array}{cr}108,3 \pm 59 & 108,8 \pm 26 \\
(53,5-185) & (92,1-147) \\
& P=0,5632\end{array}$ & $\begin{array}{rr}3,4 \pm 0,6 & 4,1 \pm 1,1 \\
(2,9-4,2) & (2,7-5,0) \\
& P=0,2574\end{array}$ \\
\hline Taekwondo & $\begin{array}{rr}158,4 \pm 32 & 138,5 \pm 14,4 \\
(122,2-186,1) & (126,8-154,6) \\
& P=0,3891 \\
\end{array}$ & $\begin{array}{rr}41,9 \pm 11 & 39 \pm 10 \\
(28,8-49) & (26,5-46,5) \\
& P=0,7684\end{array}$ & $\begin{array}{rr}99,1 \pm 37 & 77,6 \pm 12 \\
(65,9-140) & (69,2-90,3) \\
& P=0,4003\end{array}$ & $\begin{array}{cr}87,3 \pm 46 & 109,4 \pm 43 \\
(41,9-134) & (70,4-156) \\
& P=0,5793 \\
\end{array}$ & $\begin{array}{lr}4,1 \pm 2,0 & 3,7 \pm 1,2 \\
(2,5-6,4) & (2,7-5,0) \\
& P=0,8005\end{array}$ \\
\hline Natación & $\begin{array}{rr}163,6 \pm 40 & 144,4 \pm 23 \\
(132-208,9) & (118,5-189,6) \\
& P=0,3012\end{array}$ & $\begin{array}{cr}47,8 \pm 14 & 42,4 \pm 6 \\
(32,3-59,8) & (34,1-54) \\
& P=0,3330\end{array}$ & $\begin{array}{rr}88,8 \pm 21,6 & 81 \pm 24 \\
(74,1-113) & (58,6-131) \\
& P=0,6296\end{array}$ & $\begin{array}{rr}63,5 \pm 33 & 96,1 \pm 16 \\
(33,2-100) & (79,2-110) \\
& P=0,2262\end{array}$ & $\begin{array}{rr}3,8 \pm 2,2 & 3,5 \pm 0,8 \\
(2,5-6,4) & (2,6-5,0) \\
& P=0,6583\end{array}$ \\
\hline Microfútbol & $\begin{array}{r}\mathrm{F} \\
153,03 \pm 35,9(96,9-217,8)\end{array}$ & $\begin{array}{r}\mathrm{F} \\
47,35 \pm 16,6(26,1-76,1)\end{array}$ & $\begin{array}{r}F \\
89,43 \pm 29,4(56-151)\end{array}$ & $\begin{array}{r}\mathrm{F} \\
81,35 \pm 32,4(41,7-143,2)\end{array}$ & $\begin{array}{r}\mathrm{F} \\
3,34 \pm 0,7(2,2-4,2)\end{array}$ \\
\hline Halterofilia & $\begin{array}{r}\mathrm{M} \\
148,3 \pm 23,8(123,8-171,3)\end{array}$ & $\begin{array}{r}\mathrm{M} \\
33,9 \pm 5,3(30,2-40)\end{array}$ & $\begin{array}{r}\mathrm{M} \\
86,3 \pm 8,9(78,3-95,9)\end{array}$ & $\begin{array}{r}\mathrm{M} \\
140,6 \pm 61,7(69,4-177,4)\end{array}$ & $\begin{array}{r}\mathrm{M} \\
4,4 \pm 0,5(3,9-4,9)\end{array}$ \\
\hline Atletismo & $\begin{array}{rr}M \\
144,7 \pm 23(124,3-178,1)\end{array}$ & $\begin{array}{r}\mathrm{M} \\
53,6 \pm 17(38,6-78,4)\end{array}$ & $\begin{array}{r}\mathrm{M} \\
74,2 \pm 9,8(57-81)\end{array}$ & $\begin{array}{r}M \\
84,4 \pm 42(33,2-143,4)\end{array}$ & $\begin{array}{r}\mathrm{M} \\
2,8 \pm 0,4(2,3-3,2)\end{array}$ \\
\hline Triatlón & $\begin{array}{r}\mathrm{M} \\
152,7 \pm 37(117-191,1)\end{array}$ & $\begin{array}{r}\mathrm{M} \\
43,8 \pm 13(33,7-58,4)\end{array}$ & $\begin{array}{r}\mathrm{M} \\
84,6 \pm 38(48,-124,8)\end{array}$ & $\begin{array}{r}\mathrm{M} \\
121,5 \pm 63,7(52-177)\end{array}$ & $\begin{array}{r}\mathrm{M} \\
3,7 \pm 1,5(2,0-4,8)\end{array}$ \\
\hline Karate & $\begin{array}{r}\mathrm{M} \\
140,2 \pm 33(103,6-197,4)\end{array}$ & $\begin{array}{r}\mathrm{M} \\
39,2 \pm 12,9(29-62,4)\end{array}$ & $\begin{array}{r}\mathrm{M} \\
77,5 \pm 24(50,5-121)\end{array}$ & $\begin{array}{r}\mathrm{M} \\
117,7 \pm 58(66-221,7)\end{array}$ & $\begin{array}{r}\mathrm{M} \\
3,8 \pm 1,3(2,5-5,2)\end{array}$ \\
\hline Tenis de campo & $\begin{array}{r}M \\
172 \pm 28(147,9-218,6)\end{array}$ & $\begin{array}{r}\mathrm{M} \\
41 \pm 2,0 \quad(38,7-42)\end{array}$ & $\begin{array}{r}\mathrm{M} \\
106,5 \pm 28(82-152,8)\end{array}$ & $\begin{array}{r}\mathrm{M} \\
123,4 \pm 37(68-151,7)\end{array}$ & $\begin{array}{r}M \\
4,3 \pm 0,9(3,5-6)\end{array}$ \\
\hline
\end{tabular}

F: femenino. M: masculino. CT: colesterol total. HDL: lipoproteína de alta densidad. LDL: lipoproteína de baja densidad. TG: triglicéridos. IA: índice arterial.

Con respecto a las lipoproteínas de alta densidad (HDL), en promedio las mujeres se encuentran en el rango $40-60 \mathrm{mg} / \mathrm{dl}$ considerado como normal(11), sin embargo, en todas las selecciones se observa que en ellas el límite inferior está por debajo del rango normal. Con respecto a los hombres, se observa que en promedio están por debajo del rango los estudiantes deportistas de las selecciones de voleibol, fútbol, porrismo, taekwondo, karate, atletismo y halterofilia; y al observar los límites inferiores de los hombres de las demás selecciones, se observa también que están por debajo. Al comparar las concentraciones de HDL de cada selección por sexo se encontraron diferencias estadísticamente significativas en las selecciones de fútbol, porrismo y tenis de mesa.

Al observar los valores promedio de los triglicéridos es menor de $150 \mathrm{mg} / \mathrm{dl}$ indicando que los estudiantes deportistas de ambos sexos se encuentran en los límites considerados como normales, a excepción de los hombres de la selección de porrismo quienes tienen un promedio de $157,1 \mathrm{mg} / \mathrm{dl}$, es decir, con triglicéridos levemente elevados (150$199 \mathrm{mg} / \mathrm{dl}$ ). En los límites superiores se encontraron algunas personas en el rango levemente elevado y elevado, quienes pertenecían a las selecciones de fútbol, porrismo, 
tenis de mesa, karate, halterofilia y triatlón. Al hacer las comparaciones por sexo únicamente se encontró diferencia estadísticamente significativa en la selección de porrismo.

Con respecto al índice arterial, se observó que en promedio todos los estudiantes deportistas se encuentran dentro de los rangos considerados como normales según el sexo, sin embargo, se aprecia en los límites superiores de algunas selecciones, estudiantes con riesgo moderado de presentar un evento cardiovascular.

En la Tabla 2 se aprecia que el promedio de edad cronológica fue de 21 años, y el límite superior de 35 años, con respecto a la edad morfológica se encontró que en promedio las mujeres tienden a presentar una mayor edad con respecto a los hombres. Específicamente se observa que las mujeres de la selección de tenis de campo tienen en promedio una edad cronológica de 20 años, y morfológica de 33 años. Los deportistas presentaron promedios normales de IMC, sin embargo, se encontraron datos en baloncesto y tenis de campo femenino de 28,62 y $26,28 \mathrm{~kg} / \mathrm{m}^{2}$ respectivamente, indicando que hay un posible caso de sobrepeso en estos grupos de mujeres(13). Para el grupo de deportistas el ICC se encontró en promedios que oscilan entre muy bajos y bajos $(0,78$ hasta 0,91 en hombres y 0,76 hasta 0,84 en mujeres), sin embargo, en la selección de fútbol femenina se encontró que en promedio el ICC fue de 0,87 , señalando según los parámetros establecidos un alto riesgo de sufrir problemas cardiovasculares(14).

Tabla 2. Variables antropométricas por selección y sexo

\begin{tabular}{|c|c|c|c|c|c|c|}
\hline \multirow{3}{*}{ Selección } & \multicolumn{6}{|c|}{ Variables } \\
\hline & Edad crono (años) & Edad morfo (años) & Talla (m) & Masa (kg) & IMC (peso/talla ${ }^{2}$ ) & ICC \\
\hline & $\begin{array}{ll}\mathbf{F} & \mathbf{M} \\
\end{array}$ & $\begin{array}{ll}\mathbf{F} & \mathbf{M} \\
\end{array}$ & F $\quad \mathbf{M}$ & F $\quad \mathbf{M}$ & $\begin{array}{ll}\mathbf{F} & \mathbf{M} \\
\end{array}$ & $\mathbf{M}$ \\
\hline Voleibol & $\begin{array}{cr}20,5 \pm 3,1 & 20,8 \pm 3 \\
(18-27) & (18-27) \\
P=0,7544\end{array}$ & $\begin{array}{rr}27,7 \pm 4,2 & 20,58 \pm 1,7 \\
(16-31) & (12-24) \\
P=0,0000\end{array}$ & $\begin{array}{lr}1,62 \pm 0,01 & 1,73 \pm 0,1 \\
(1,5-1,7) & (1,6-1,8) \\
& P=0,0019\end{array}$ & $\begin{array}{lr}55,8 \pm 4,7 & 73,2 \pm 8,3 \\
(48-62) & (63-85) \\
& P=0,0000\end{array}$ & $\begin{array}{cc}21,10 \pm 2 & 22,66 \pm 6,7 \\
(18-25) & (3,8-27,5) \\
& P=0,4948\end{array}$ & $\begin{array}{rr}0,83 \pm 0,0 & 0,86 \pm 0,0 \\
(0,7-0,9) & (0,8-0,9) \\
P=0,0784\end{array}$ \\
\hline Fútbol & $\begin{array}{rr}20,68 \pm 2,1 & 22,12 \pm 2,3 \\
(18-25) & (18-26) \\
& P=0,0562\end{array}$ & $\begin{array}{rr}29,2 \pm 5,5 & 20,7 \pm 2,7 \\
(15-45) & (5-27) \\
& P=0,000\end{array}$ & $\begin{array}{rr}1,56 \pm 0,0 & 1,73 \pm 0,0 \\
(1,45-1,66) & (1,63-1,85) \\
P=0,0000\end{array}$ & $\begin{array}{rr}56,1 \pm 8,3 & 67,1 \pm 6,0 \\
(41,2-71)(56,7-74,6) \\
P=0,0000\end{array}$ & $\begin{array}{rr}23,37 \pm 3,3 & 22,31 \pm 2,1 \\
(16,5-28,8) & (18,6-25,5) \\
& P=0,2764\end{array}$ & $\begin{array}{cr}0,87 \pm 0,0 & 0,78 \pm 0,1 \\
(0,7-0,9) & (0,2-0,8) \\
& P=0,0171\end{array}$ \\
\hline Baloncesto & $\begin{array}{r}20,57 \pm 1,7 \quad 20,33 \pm 1,9 \\
(18-26) \quad(17-23) \\
P=0,8017\end{array}$ & $\begin{array}{r}21,40 \pm 3,0 \quad 23,27 \pm 3,9 \\
(17-26) \quad(18-29) \\
P=0,3153\end{array}$ & $\begin{array}{rr}1,60 \pm 0,0 & 1,78 \pm 0,0 \\
(1,56-1,68) & (1,73- \\
1,88) \\
\mathrm{P}=0,0000 \\
\end{array}$ & $\begin{array}{rr}55,9 \pm 9,3 & 76,96 \pm 12,1 \\
(44,8-74,5) & (60-99,3) \\
& P=0,0020\end{array}$ & $\begin{array}{lr}28,62 \pm 2,1 & 20,95 \pm 2,0 \\
(26,7-32,7) & (18,8-24) \\
& P=0,0000\end{array}$ & $\begin{array}{rr}0,77 \pm 0,0 & 0,87 \pm 0,0 \\
(0,73-0,82) & (0,81-0,9) \\
& P=0,0002\end{array}$ \\
\hline Porrismo & $\begin{array}{r}22,66 \pm 3,7 \quad 21,5 \pm 3,8 \\
(18-27) \quad(18-29) \\
P=0,5454\end{array}$ & $\begin{array}{rr}27,03 \pm 1,6 & 24,61 \pm 5,1 \\
(24,3-28) & (18,6-35,2) \\
P=0,2825\end{array}$ & $\begin{array}{rr}1,51 \pm 0,0 \quad 1,68 \pm 0,0 \\
(1,45-1,65) & (1,62- \\
1,83) \\
P=0,0000 \\
\end{array}$ & $\begin{array}{rr}46,78 \pm 3,7 & 69,0 \pm 10,0 \\
(42,5-51,8) & (54-90,5) \\
& P=0,0000\end{array}$ & $\begin{array}{rr}20,65 \pm 0,7 & 23,10 \pm 2,6 \\
(19,9-22,0) & (19,3-27,8) \\
& P=0,04596\end{array}$ & $\begin{array}{rr}0,76 \pm 0,0 & 0,85 \pm 0,0 \\
(0,68-0,81) & (0,79-0,9) \\
& P=0,00210\end{array}$ \\
\hline Squash & $\begin{array}{rr}20,5 \pm 2,6 & 21,71 \pm 6,2 \\
(18-24) & (18-35) \\
P=0,07250\end{array}$ & $\begin{array}{rr}28,4 \pm 2,1 & 20,57 \pm 3,9 \\
(25,3-30) & (16,7-29,0) \\
& P=0,00572\end{array}$ & $\begin{array}{rr}1,56 \pm 0,0 & 1,69 \pm 0,0 \\
(1,47-1,63) & (1,65- \\
1,79) \\
P=0,0030 \\
\end{array}$ & $\begin{array}{rr}55,62 \pm 8,0 & 62,5 \pm 4,3 \\
(43,8-61,1) & (56-69,0) \\
P=0,09301\end{array}$ & $\begin{array}{rr}22,52 \pm 1,6 & 21,64 \pm 1,6 \\
(20,2-24,1) & (19,3-24,4) \\
P=0,4142\end{array}$ & $\begin{array}{rr}0,77 \pm 0,0 & 0,81 \pm 0,0 \\
(0,72-0,82) & (0,77-0,85) \\
P=0,0786\end{array}$ \\
\hline Tenis de mesa & $\begin{array}{rr}20,75 \pm 1,7 & 21,0 \pm 3,0 \\
(19-23) & (18-25) \\
P=0,8859\end{array}$ & $\begin{array}{rr}28,12 \pm 0,0 & 20,26 \pm 0,0 \\
(26,6-29) & (17,6-23,6) \\
& P=0,00014\end{array}$ & $\begin{array}{rr}1,58 \pm 0,0 & 1,69 \pm 0,0 \\
(1,55-1,64) & (1,65- \\
1,79) \\
P=0,0058 \\
\end{array}$ & $\begin{array}{rr}55,32 \pm 5,7 & 67,6 \pm 13,5 \\
(48,8-63,5) & (52-87,8) \\
P=0,1314\end{array}$ & $\begin{array}{rr}22,19 \pm 2,6 & 22,93 \pm 3,1 \\
(20,1-26-06) & (19,3-28,0) \\
& P=0,7128\end{array}$ & $\begin{array}{rr}0,84 \pm 0,0 & 0,85 \pm 0,0 \\
(0,8-0,87) & (0,8-0,9) \\
& P=0,2383\end{array}$ \\
\hline Taekwondo & $\begin{array}{r}18,33 \pm 0,5 \quad 20,33 \pm 1,1 \\
(18-19) \quad(19-21) \\
P=0,0550\end{array}$ & $\begin{array}{rr}27,1 \pm 2,0 & 19,80 \pm 2,2 \\
(25,3-29,3) & (17,7- \\
22,2) \\
\mathrm{P}=0,0144 \\
\end{array}$ & $\begin{array}{rr}1,54 \pm 0,0 & 1,67 \pm 0,0 \\
(1,46-1,64) & (1,59- \\
1,74) \\
P=0,1304 \\
\end{array}$ & $\begin{array}{cc}52,4 \pm 8,1 & 65,86 \pm 7,9 \\
(46,0-61,6) & (58,1-74) \\
& P=0,1102\end{array}$ & $\begin{array}{rr}21,77 \pm 1,8 & 23,38 \pm 0,9 \\
(19,6-22,9) & (22,7-24,4) \\
P=0,2486\end{array}$ & $\begin{array}{rr}0,83 \pm 0,0 & 0,82 \pm 0,0 \\
(0,82-0,84) & (0,8-0,86) \\
& P=0,6560\end{array}$ \\
\hline Natación & $\begin{array}{rr}21,0 \pm 5,0 & 21,0 \pm 2,4 \\
(18-26) & (18-26) \\
P=0,9800\end{array}$ & $\begin{array}{rr}22,96 \pm 7,8 \quad 20,50 \pm 1,3 \\
(16,1-31,1) & (18,7- \\
23,3) \\
P=0,3205\end{array}$ & $\begin{array}{rr}1,62 \pm 0,0 & 1,73 \pm 0,0 \\
(1,61-1,66) & (1,64- \\
1,81) \\
P=0,0062 \\
\end{array}$ & $\begin{array}{rr}64,2 \pm 5,0 & 69,7 \pm 8,4 \\
58,9-69,0) & (56,1-81) \\
& P=0,3160\end{array}$ & $\begin{array}{r}24,18 \pm 2,423,06 \pm 1,6 \\
(22,3-26,9)(20,8-25,1) \\
P=0,3593\end{array}$ & $\begin{array}{cr}0,77 \pm 0,1 & 0,79 \pm 0,1 \\
(0,77-0,77) & (0,5-0,87) \\
& P=0,0892\end{array}$ \\
\hline Microfútbol & $\begin{array}{r}F \\
20,8 \pm 3,2(18-25)\end{array}$ & $\begin{array}{r}\mathrm{F} \\
28,2 \pm 2,7(25-33)\end{array}$ & $\begin{array}{r}\mathrm{F} \\
1,53 \pm 0,0(1,48-1,59)\end{array}$ & $\begin{array}{r}F \\
53,42 \pm 9,5(41,6-70,2)\end{array}$ & $\begin{array}{r}F \\
22,62 \pm 3,4(19,49-29,6)\end{array}$ & $\begin{array}{r}F \\
0,83 \pm 0,0(0,74-0,99)\end{array}$ \\
\hline Halterofilia & $\begin{array}{r}M \\
23,33 \pm 6,6(19-31)\end{array}$ & $\begin{array}{r}\mathrm{M} \\
27,66 \pm 5,5(24,0-34,1)\end{array}$ & $\begin{array}{r}\mathrm{M} \\
1,71 \pm 0,0 \quad(1,66-1,78)\end{array}$ & $\begin{array}{r}\mathrm{M} \\
96,2 \pm 8,9(88,6-106)\end{array}$ & $\begin{array}{r}\mathrm{M} \\
33,78 \pm 2,1(30,0-34,1)\end{array}$ & $\begin{array}{r}\mathrm{M} \\
0,91 \pm 0,0(0,88-0,94)\end{array}$ \\
\hline Atletismo & $\begin{array}{r}\mathrm{M} \\
21,0 \pm 1,5(19-23) \\
\end{array}$ & $\begin{array}{r}\mathrm{M} \\
19,4 \pm 1,4(17,2-20,8) \\
\end{array}$ & $\begin{array}{r}\mathrm{M} \\
1,71 \pm 0,0(1,62-1,86) \\
\end{array}$ & $\begin{array}{r}\mathrm{M} \\
61,4 \pm 5,5(55,3-69-3) \\
\end{array}$ & $\begin{array}{r}\mathrm{M} \\
20,79 \pm 0,91(19,89-22,14)\end{array}$ & $\begin{array}{r}\mathrm{M} \\
0,80 \pm 0,0(0,77-0,83)\end{array}$ \\
\hline Triatlón & $\begin{array}{r}\mathrm{M} \\
21,0 \pm 5,5(18-26)\end{array}$ & $\begin{array}{r}\mathrm{M} \\
19,73 \pm 3,5(16-23)\end{array}$ & $\begin{array}{r}\mathrm{M} \\
1,73 \pm 0,0(1,69-1,76)\end{array}$ & $\begin{array}{r}\mathrm{M} \\
66,73 \pm 4,7(62,1-71,9)\end{array}$ & $\begin{array}{r}\mathrm{M} \\
22,16 \pm 0,9(21,4-23,2)\end{array}$ & $\begin{array}{r}\mathrm{M} \\
0,85 \pm 0,0(0,82-0,88)\end{array}$ \\
\hline Karate & $\begin{array}{r}\mathrm{M} \\
22,66 \pm 2,7(20-27)\end{array}$ & $\begin{array}{r}\mathrm{M} \\
21,33 \pm 1,4(19,6-23,5) \\
\end{array}$ & $\begin{array}{r}\mathrm{M} \\
1,69 \pm 0,0(1,62-1,8) \\
\end{array}$ & $\begin{array}{r}\mathrm{M} \\
64,05 \pm 3,6(58,5-68,1)\end{array}$ & $\begin{array}{r}\mathrm{M} \\
22,35 \pm 0,9(20,6-23,2)\end{array}$ & $\begin{array}{r}\mathrm{M} \\
0,87 \pm 0,0(0,81-0,98) \\
\end{array}$ \\
\hline $\begin{array}{l}\text { Tenis de } \\
\text { campo }\end{array}$ & $\begin{array}{r}M \\
M \\
19,8 \pm 1,3(18-21)\end{array}$ & $\begin{array}{r}M \\
20,78 \pm 1,5(19,2-22,5)\end{array}$ & $\begin{array}{r}\mathrm{M} \\
1,69 \pm 0,0(1,6-1,75)\end{array}$ & $\begin{array}{r}M \\
68,94 \pm 14,1(57,4-0,4)\end{array}$ & $\begin{array}{r}M \\
M \\
23,81 \pm 3,6(20,9-29,7)\end{array}$ & $\begin{array}{r}\mathrm{M} \\
0,81 \pm 0,0(0,75-0,96)\end{array}$ \\
\hline
\end{tabular}

IMC: Índice de Masa Corporal. ICC: Índice Cintura Cadera. 
Con respecto a la composición corporal se encontró que las mujeres de baloncesto y tenis de mesa manejan porcentajes de grasa promedio de 30,62 y 32,4 respectivamente (Tabla 3 ). En porrismo, se encontró un promedio de $22,73 \%$ de grasa y de músculo de $47,33 \%$, estando dentro los rangos normales; en el caso de los hombres, los porcentajes oscilaron entre el 20 y el $25 \%$. Los deportes individuales como el triatlón, karate y taekwondo presentan promedios entre 21 y $22 \%$ de grasa y en disciplinas de conjunto el voleibol y el baloncesto con el 22 y $23 \%$.

Tabla 3. Variables de composición corporal por selección y sexo

\begin{tabular}{|c|c|c|c|c|c|}
\hline \multirow[t]{3}{*}{ Selección } & \multicolumn{5}{|c|}{ Variables } \\
\hline & $\%$ Muscular & \% Adiposo & \% Óseo & $\%$ Residual & $\%$ Piel \\
\hline & $\begin{array}{ll}\mathbf{F} & \mathbf{M}\end{array}$ & F $\quad$ M & M & F $\quad$ M & M \\
\hline Voleibol & $\begin{array}{r}40,43 \pm 4,148,35 \pm 1,7 \\
(31,6-45,0)(45,3-50,6) \\
P=0,0000\end{array}$ & $\begin{array}{rr}29,80 \pm 5,3 & 22,18 \pm 1,8 \\
(25,1-43,6)(19,18-24,84) \\
P=0,0000\end{array}$ & $\begin{array}{r}11,24 \pm 9,1 \quad 11,00 \pm 0,8 \\
(9,11-14,67)(9,72-12,16) \\
P=0,6995\end{array}$ & 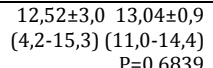 & $\begin{array}{r}6,09 \pm 0,75,40 \pm 0,2 \\
(5,24-7,73)(5,02-5,77) \\
P=0-01662\end{array}$ \\
\hline Futbol & $\begin{array}{r}38,70 \pm 5,8 \quad 43,07 \pm 4,4 \\
(20,15-45,18)(30,37-47,5) \\
P=0,0165\end{array}$ & $\begin{array}{rr}29,84 \pm 4,7 & 25,64 \pm 3,6 \\
(13,33-35,5)(15,66-30,39) \\
P=0,0054 \\
\end{array}$ & $\begin{array}{rr}12,87 \pm 10,8 & 12,34 \pm 7,3 \\
(8,25-53,14)(6,71-39,6) \\
P=0,8656\end{array}$ & $\begin{array}{r}13,08 \pm 1,6 \quad 12,91 \pm 0,9 \\
(7,3-15,5)(10,7-14,7) \\
P=0,7225 \\
\end{array}$ & $\begin{array}{rr}5,48 \pm 0,9 & 5,74 \pm 0,7 \\
(3,7-6,97)(3,68-6,84) & (\mathrm{P}=0,3516 \\
\end{array}$ \\
\hline Baloncesto & $\begin{array}{rr}39,51 \pm 3,7 & 45,07 \pm 3,8 \\
(35,14-46,45)(39,0-53,12) \\
P=0,01111\end{array}$ & $\begin{array}{rr}30,62 \pm 3,4 & 23,58 \pm 3,3 \\
(28,8-33,68)(17,63-29,71) \\
P=0,0010\end{array}$ & $\begin{array}{rr}11,40 \pm 1,4 & 11,62 \pm 1,2 \\
(9,49-12,87)(10,25,13,9) \\
P=0,7556\end{array}$ & $\begin{array}{rr}12,45 \pm 1,2 & 14,23 \pm 0,9 \\
(11,1-14,1) & (13-15,7) \\
& P=0,0057\end{array}$ & $\begin{array}{rr}6,01 \pm 0,7 & 5,46 \pm 0,6 \\
(5,16-7,48)(4,65-6,63) \\
P=0,1657\end{array}$ \\
\hline Squash & $\begin{array}{r}40,43 \pm 4,1 \quad 46,29 \pm 1,3 \\
(31,6-45,0)(44,73-48,3) \\
\mathrm{P}=0,0031\end{array}$ & $\begin{array}{rr}28,64 \pm 1,2 & 24,78 \pm 1,6 \\
(27,5-30,37)(21,52-26-66) \\
P=0,0029\end{array}$ & $\begin{array}{r}10,70 \pm 0,3 \quad 11,25 \pm 1,5 \\
(10,21-11,13)(8,63-13,8) \\
P=0,5077\end{array}$ & $\begin{array}{r}12,25 \pm 0,912,28 \pm 0,8 \\
(11,4-13,6)(10-13,4) \\
P=0,9612\end{array}$ & $\begin{array}{rr}5,63 \pm 0,4 & 5,80 \pm 0,3 \\
(5,31-6,36)(5,37-6,37) \\
P=0,5411\end{array}$ \\
\hline Tenis de mesa & $\begin{array}{r}37,97 \pm 2,1 \quad 42,72 \pm 3,5 \\
(36,41-31,14)(38,2-46,5) \\
P=0,0465\end{array}$ & $\begin{array}{rr}32,4 \pm 0,8 & 27,05 \pm 3,2 \\
(31,53-33,3)(24,34-30,94) \\
P=0,0124\end{array}$ & $\begin{array}{rr}9,91 \pm 0,4 & 11,51 \pm 1,6 \\
(9,33-10,51)(9,25-13,87) & \left(\begin{array}{l}9 \\
P\end{array}\right), 09487\end{array}$ & $\begin{array}{r}14,21 \pm 1,2 \quad 13,43 \pm 0,4 \\
(12,6-15)(12,7-13,9) \\
P=0,1846\end{array}$ & $\begin{array}{rr}5,50 \pm 0,7 & 5,44 \pm 0,5 \\
(4,51-6,1)(4,44-5,98) \\
P=0,885\end{array}$ \\
\hline Taekwondo & $\begin{array}{rr}39,55 \pm 3,0 & 47,75 \pm 0,4 \\
(36,16-42)(47,47-48,27) \\
P=0,0102\end{array}$ & $\begin{array}{rr}29,08 \pm 2,7 & 22,12 \pm 1,1 \\
(26-31,17)(20,85-23,17) \\
P=0,0150\end{array}$ & $\begin{array}{rr}11,19 \pm 0,5 & 10,80 \pm 2,3 \\
(10,8-11,85)(8,15-12,41) \\
P=0,7947\end{array}$ & $\begin{array}{rr}14,27 \pm 0,8 & 13,69 \pm 1,7 \\
(13,4-15)(12,1-15-6) \\
P=0,6361\end{array}$ & $\begin{array}{r}5,91 \pm 0,5 \quad 5,62 \pm 0,3 \\
(5,42-6,52)(5,35-5,95) \\
P=0,4790\end{array}$ \\
\hline Porrismo & $\begin{array}{rr}47,33 \pm 0,9 & 47,13 \pm 4,2 \\
(44,46-52,45)(36,5-53,28) \\
\mathrm{P}=0,9176 \\
\end{array}$ & $\begin{array}{rr}22,73 \pm 2,5 & 23,56 \pm 3,5 \\
(2,22-27,07)(19,11-30,89) \\
P=0,6160 \\
\end{array}$ & $\begin{array}{r}10,98 \pm 1,111,11 \pm 1,3 \\
(8,99-12,36)(8,28-12,85) \\
P=0,8412\end{array}$ & $\begin{array}{r}12,74 \pm 1,1 \quad 12,82 \pm 1,0 \\
(11-14,4)(10,69-4,3) \\
P=0,8736\end{array}$ & $\begin{array}{r}6,20 \pm 0,2 \quad 5,36 \pm 0,6 \\
(5,73-6,55)(4,59-6,49) \\
P=0,0058\end{array}$ \\
\hline Natación & $\begin{array}{rr}43,27 \pm 3,8 & 45,68 \pm 4,3 \\
(38,9-46-1)(40,76-54-78) \\
P=0,4120\end{array}$ & $\begin{array}{rr}27,62 \pm 3,9 & 22,64 \pm 3,4 \\
(24,7-32,15)(16,22-27,73) \\
P=0,0580\end{array}$ & $\begin{array}{rr}10,82 \pm 0,8 & 12,98 \pm 1,7 \\
(10,12-11,7)(10,16-16,5) \\
P=0,0718\end{array}$ & $\begin{array}{r}12,84 \pm 0,4 \quad 13,11 \pm 2,5 \\
(12,4-13,3)(6,6,16,4) \\
P=0,8609\end{array}$ & $\begin{array}{rr}5,44 \pm 0,7 & 5,58 \pm 0,6 \\
(4,76-6,16)(4,89-6,84) \\
P=0,7307\end{array}$ \\
\hline Microfútbol & $\begin{array}{r}F \\
41,27 \pm 3,4(33,82-44,79)\end{array}$ & $\begin{array}{r}\mathrm{F} \\
30,29 \pm 2,4(27,5-34,65)\end{array}$ & $\begin{array}{r}F \\
9,64 \pm 1,8(6,94-12,4)\end{array}$ & $\begin{array}{r}F \\
13,11 \pm 1,3(11,6-15,3)\end{array}$ & $\begin{array}{r}\mathrm{F} \\
5,66 \pm 0,7(4,54-6,83)\end{array}$ \\
\hline Heterofilia & $\begin{array}{r}\mathrm{M} \\
48,63 \pm 4,1(44,03-51,97)\end{array}$ & $\begin{array}{r}\mathrm{M} \\
20,68 \pm 3,2(18,13-24,33)\end{array}$ & $\begin{array}{r}\mathrm{M} \\
12,14 \pm 0,6(11,34-12,6)\end{array}$ & $\begin{array}{r}\mathrm{M} \\
14,34 \pm 1,2(12,8-15,2)\end{array}$ & $\begin{array}{r}\mathrm{M} \\
4,20 \pm 0,0(4,14-4,25)\end{array}$ \\
\hline Atletismo & $\begin{array}{r}\mathrm{M} \\
44,91 \pm 4,4(41,4-52-69)\end{array}$ & $\begin{array}{r}\mathrm{M} \\
23,36 \pm 4,0(17,07-27,66)\end{array}$ & $\begin{array}{r}\mathrm{M} \\
11,73 \pm 1,0(10,48-13,15)\end{array}$ & $\begin{array}{r}\mathrm{M} \\
13,83 \pm 0,6(12,8-14,3)\end{array}$ & $\begin{array}{r}\mathrm{M} \\
6,14 \pm 0,3(5,72-6,64)\end{array}$ \\
\hline Triatlón & $\begin{array}{r}M \\
43,33 \pm 5,0(39,74-49-13)\end{array}$ & $\begin{array}{r}M \\
21,58 \pm 5,6(15,09-25,3)\end{array}$ & $\begin{array}{r}M \\
14,3 \pm 0,9(13,72-15,34)\end{array}$ & $\begin{array}{r}M \\
14,89 \pm 0,7(14,3-15,6)\end{array}$ & $\begin{array}{r}M \\
5,9 \pm 0,4(5,57-6,39)\end{array}$ \\
\hline Karate & $\begin{array}{r}\mathrm{M} \\
47,67 \pm 0,9(46,34-48,51\end{array}$ & $\begin{array}{r}\mathrm{M} \\
20,47 \pm 2,2(16,71-23,42)\end{array}$ & $\begin{array}{r}M \\
11,79 \pm 1,4(9,18-13,2)\end{array}$ & $\begin{array}{r}\mathrm{M} \\
14,36 \pm 1,5(12,4-16,9)\end{array}$ & $\begin{array}{r}\mathrm{M} \\
5,69 \pm 0,2(5,39-6,1)\end{array}$ \\
\hline Tenis de campo & $\begin{array}{r}M \\
45,57 \pm 3,8(39,46-49-67)\end{array}$ & $\begin{array}{r}\mathrm{M} \\
24,09 \pm 4,9(20,54-32,67)\end{array}$ & $\begin{array}{r}\mathrm{M} \\
12,18 \pm 1,3(10,85-14,3)\end{array}$ & $\begin{array}{r}M \\
12,69 \pm 1,2(11,4-14,6)\end{array}$ & $\begin{array}{r}\mathrm{M} \\
5,46 \pm 0,6(4,35-6,07)\end{array}$ \\
\hline
\end{tabular}

En relación a las variables de la condición física se encontró los mejores promedios en las mujeres de taekwondo $(15,56 \mathrm{~cm})$, los hombres de porrismo $(16,36 \mathrm{~cm})$ y los de karate $(18,45 \mathrm{~cm})$, por el contrario, triatlón $(6,5 \mathrm{~cm})$, las mujeres de natación $(7,7 \mathrm{~cm}) \quad \mathrm{y}$ baloncesto femenino $(6,12 \mathrm{~cm})$, fueron los que menores resultados obtuvieron en la evaluación. Con respecto a la fuerza los hombres de la selección de karate presentaron en promedio el mejor resultado, seguido a estos los hombres de porrismo y las selecciones masculinas de baloncesto y voleibol.

Frente a la variable velocidad los deportistas de la Universidad del Quindío obtuvieron promedios que van desde 3,61 s por los hombres de fútbol y
3,85 s por las mujeres de tenis de mesa, mientras que los hombres de triatlón con 5,55s y 7,04s en mujeres de karate, quienes requirieron más tiempo para recorrer los $30 \mathrm{~m}$. La selección de atletismo alcanzo tiempo promedio de $3,66 \mathrm{~s}$, sin embargo, no todos eran velocistas, lo que no permitió que tuvieran el mejor promedio de velocidad.

La evaluación de la resistencia se basó en la posibilidad de estimar a través de un test de potencia aeróbica máxima (Test Legger)(15), el consumo máximo de oxígeno y de esa forma perfilarlos en su condición. Este test evidenció que en promedio los hombres de triatlón, atletismo, baloncesto y taekwondo alcanzaron el 
nivel 10; voleibol, futbol, squash, karate y natación el nivel 9; tenis de mesa, tenis de campo, porrismo el nivel 8 y halterofilia el nivel 7. En las mujeres, la selección de voleibol obtuvo el más alto nivel (nivel 7), mientras que deportes individuales como squash, tenis de mesa, de campo, taekwondo fueron los más bajos (nivel 5). Estos niveles se representan de acuerdo a la fórmula $\mathrm{VO}_{2 \max }$ (Tabla 4), el mayor $\mathrm{VO}_{2 \max }$ alcanzado por los hombres fue de atletismo y triatlón con 40,31 y $38,77 \mathrm{ml} / \mathrm{kg} / \mathrm{min}$ respectivamente y el menor fue el de halterofilia con $21,56 \mathrm{ml} / \mathrm{kg} / \mathrm{min}$; para el caso de las mujeres, el máximo promedio fue de $22,08 \mathrm{ml} / \mathrm{kg} / \mathrm{min}$ alcanzado por voleibol y el menor por tenis de campo con $9,8 \mathrm{ml} / \mathrm{kg} / \mathrm{min}$ ubicando a todas las disciplinas deportivas femeninas en rangos de medio bajo y pobre.

Tabla 4. Variables de condición física por selección y sexo

\begin{tabular}{|c|c|c|c|c|c|}
\hline \multirow{3}{*}{ Selección } & \multicolumn{5}{|c|}{ Variables } \\
\hline & Flexibilidad (cm) & Fuerza (mts) & Velocidad (segundos) & Resistencia (nivel) & $\mathrm{VO}_{2 \max }(\mathrm{ml} / \mathrm{kg} / \mathrm{min})$ \\
\hline & M & $\mathbf{F} \quad \mathbf{M}$ & F $\quad$ M & M & M \\
\hline Voleibol & $\begin{array}{r}13,15 \pm 8,211,58 \pm 8,5 \\
(--4,4-23,15)(--3,9-22,8) \\
P=0,6736\end{array}$ & $\begin{array}{rr}1,88 \pm 0,1 & 2,31 \pm 0,1 \\
(1,65-2,17)(2,04-2,67) \\
P=0,0000\end{array}$ & $\begin{array}{r}4,31 \pm 0,4 \quad 3,64 \pm 0,2 \\
(3,72-5,14)(3,15-4,17) \\
P=0,0003\end{array}$ & $\begin{array}{r}7,0 \pm 1,6 \quad 9,0 \pm 1,3 \\
(6-11)(8-12) \\
P=0,0002\end{array}$ & $\begin{array}{r}22,08 \pm 9,838,60 \pm 8,0 \\
(10,9-45,0)(27,42-50,86) \\
P=0,0004\end{array}$ \\
\hline Futbol & $\begin{array}{rr}8,88 \pm 5,2 & 3,65 \pm 7,2 \\
(--0,25-20,15)(--6,5-21,5) \\
P=0,01394\end{array}$ & $\begin{array}{r}1,58 \pm 0,1 \quad 2,16 \pm 0,1 \\
(1,27-1,86)(1,62-2,34) \\
P=0,0000\end{array}$ & $\begin{array}{r}4,40 \pm 0,2 \quad 3,61 \pm 0,1 \\
(4,06-4,82)(3,36-3,88) \\
P=0,0000\end{array}$ & $\begin{array}{r}6,0 \pm 1,19,0 \pm 1,3 \\
(5-9)(7-12) \\
P=0,0000\end{array}$ & $\begin{array}{r}19,31 \pm 6,837,21 \pm 7,8 \\
(9,84-33,28)(21,26-50,86) \\
P=0,0000\end{array}$ \\
\hline Baloncesto & 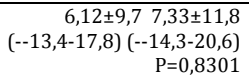 & $\begin{array}{rr}1,59 \pm 0,1 & 2,31 \pm 0,2 \\
(1,38-1,34)(1,99-2,61) \\
P=0,0000\end{array}$ & $\begin{array}{r}4,53 \pm 0,3 \quad 3,53 \pm 0,1 \\
(4,16-5,11)(3,12-3,7) \\
P=0,0000\end{array}$ & $\begin{array}{r}6,0 \pm 0,710,0 \pm 1,6 \\
(5-7)(7-13) \\
P=0,0002\end{array}$ & $\begin{array}{rr}19,88 \pm 4,4 & 38,47 \pm 9,9 \\
(9,84-21,26)(21,56-56,72) \\
P=0,0004\end{array}$ \\
\hline Squash & $\begin{array}{rr}12,42 \pm 3,7 & 11,81 \pm 6,2 \\
(7,25-15,75)(3,25-20,75) \\
P=0,8642\end{array}$ & $\begin{array}{rr}1,54 \pm 0,1 & 2,21 \pm 0,2 \\
(1,42-1,68)(1,87-2,42) \\
P=0,0001\end{array}$ & $\begin{array}{r}4,82 \pm 0,2 \quad 3,65 \pm 0,2 \\
(4,52-5,04)(3,28-3,87) \\
P=0,0000\end{array}$ & $\begin{array}{r}5,0 \pm 1,0 \quad 9,0 \pm 1,2 \\
(4-6)(8-11) \\
P=0,0005\end{array}$ & $\begin{array}{rr}15,27 \pm 0,8 & 29,65 \pm 11,0) \\
(13,98-15,7) & (18,77-45,0) \\
P=0,0041\end{array}$ \\
\hline Tenis de mesa & $\begin{array}{rr}8,07 \pm 8,3 & 11,81 \pm 6,2 \\
(4,5-20,8)(3,25-20,75) \\
P=0,9941\end{array}$ & $\begin{array}{rr}2,02 \pm 0,2 & 2,21 \pm 0,2 \\
(1,5-2,25)(1,87-2,42) \\
P=0,0078\end{array}$ & $\begin{array}{r}3,85 \pm 0,1 \quad 3,65 \pm 0,2 \\
(3,7-4,03)(3,28-3,87) \\
P=0,0000\end{array}$ & $\begin{array}{r}5,0 \pm 0,58,0 \pm 1,3 \\
(5-6)(8-11) \\
P=0,0011\end{array}$ & $\begin{array}{r}14,23 \pm 2,933,28 \pm 8,2 \\
(9,84-15,7)(27,42-45,0) \\
P=0,0014\end{array}$ \\
\hline Taekwondo & $\begin{array}{rr}15,56 \pm 3,9 & 10,48 \pm 6,3 \\
(12,1-19,85)(3,7-16-25) \\
P=0,3033\end{array}$ & $\begin{array}{rr}1,60 \pm 0,1 & 2,24 \pm 0,0 \\
(1,48-1,68) & (2,2-2,27) \\
P=0,0006\end{array}$ & $\begin{array}{r}4,62 \pm 0,1 \quad 3,73 \pm 0,1 \\
(4,52-4,79)(3,66-3,85) \\
P=0,0010\end{array}$ & $\begin{array}{r}5,0 \pm 1,1 \quad 10,0 \pm 1,1 \\
(4-6)(9-11) \\
P=0,0060\end{array}$ & $\begin{array}{r}15,12 \pm 0,941,09 \pm 6,7 \\
(13,98-15,7)(33,28-45,0) \\
P=0,0027\end{array}$ \\
\hline Porrismo & $\begin{array}{rr}16,36 \pm 6,4 & 14,46 \pm 7,0 \\
(8,3-24,6) & (1,8-24,6) \\
& P=0,0765\end{array}$ & $\begin{array}{r}1,76 \pm 0,1 \quad 2,30 \pm 0,2 \\
(1,6-1,93)(1,94-2,72) \\
P=0,0000\end{array}$ & $\begin{array}{r}4,68 \pm 0,8 \quad 3,84 \pm 0,2 \\
(3,97-6,02)(3,39-4,21) \\
P=0,0041\end{array}$ & $\begin{array}{r}6,0 \pm 0,7 \quad 8,0 \pm, 8 \\
(6-8)(6-11) \\
P=0,1536\end{array}$ & $\begin{array}{r}18,25 \pm 3,0 \quad 25,91 \pm 10,7 \\
(15,7-21,56)(15,7-45,0) \\
P=0,1457\end{array}$ \\
\hline Natación & $\begin{array}{rr}7,7 \pm 1,5 & 10,38 \pm 9,9 \\
(6,0-9,0)(--4,45-26,85) \\
P=0,6620\end{array}$ & $\begin{array}{rr}1,55 \pm 0,0 & 2,21 \pm 0,3 \\
(1,52-1,58)(1,81-2,96) \\
P=0,0057\end{array}$ & $\begin{array}{r}4,95 \pm 0,5 \quad 3,75 \pm 0,2 \\
(4,45-5,54)(3,43-4,04) \\
P=0,0000\end{array}$ & $\begin{array}{r}6,0 \pm 1,09,0 \pm 1,5 \\
(5-7)(7-11) \\
P=0,0023\end{array}$ & $\begin{array}{r}15,7 \pm 5,8 \quad 37,96 \pm 9,0 \\
(9,84-21,56)(21,46-45,0) \\
P=0,0023\end{array}$ \\
\hline Microfútbol & $\begin{array}{r}\mathrm{F} \\
11,70 \pm 5,2(3,65-20,55)\end{array}$ & $\begin{array}{r}\mathrm{F} \\
1,54 \pm 0,11(1,39-1,73)\end{array}$ & $\begin{array}{r}\mathrm{F} \\
4,52 \pm 0,3(4,12-4,99)\end{array}$ & $\begin{array}{r}\mathrm{F} \\
6,0(5,0-8,0)\end{array}$ & $\begin{array}{r}\mathrm{F} \\
17,16 \pm 5,1(9,84-27,4)\end{array}$ \\
\hline Heterofilia & $\begin{array}{r}\mathrm{M} \\
8,83 \pm 9,2(1,35-19,15)\end{array}$ & $\begin{array}{r}\mathrm{M} \\
2,09 \pm 0,3(1,7-2,43)\end{array}$ & $\begin{array}{r}\mathrm{M} \\
4,02 \pm 0,2(3,79-4,31)\end{array}$ & $\begin{array}{r}\mathrm{M} \\
7,0 \pm 1,0(6-8)\end{array}$ & $\begin{array}{r}\mathrm{M} \\
21,56 \pm 5,8(15,7-27,42)\end{array}$ \\
\hline Atletismo & $\begin{array}{r}\mathrm{M} \\
11,19 \pm 5,1(2,9-15,2)\end{array}$ & $\begin{array}{r}\mathrm{M} \\
2,22 \pm 0,6(1,62-3,23)\end{array}$ & $\begin{array}{r}\mathrm{M} \\
3,66 \pm 0,3(3,36-4,22)\end{array}$ & $\begin{array}{r}\mathrm{M} \\
10 \pm 1,4(8-12)\end{array}$ & $\begin{array}{r}\mathrm{M} \\
40,31 \pm 8,6(27,42-50,86)\end{array}$ \\
\hline Triatlón & $\begin{array}{r}\mathrm{M} \\
6,5 \pm 11,7(--5,0-18,5)\end{array}$ & $\begin{array}{r}\mathrm{M} \\
2,13 \pm 0,2(1,92-2,33)\end{array}$ & $\begin{array}{r}\mathrm{M} \\
3,89 \pm 0,3(3,48-4,24)\end{array}$ & $\begin{array}{r}M \\
10,0 \pm 1,7(9-12)\end{array}$ & $38,77 \pm 10,4(32,18-50,86)$ \\
\hline Karate & $\begin{array}{r}\mathrm{M} \\
18,45 \pm 2,5(14,55-21,2)\end{array}$ & $\begin{array}{r}\mathrm{M} \\
2,35 \pm 0,1(2,12-2,51)\end{array}$ & $\begin{array}{r}M \\
3,62 \pm 0,1(3,47-3,81)\end{array}$ & $\begin{array}{r}\mathrm{M} \\
9,0 \pm 1,6(7-11)\end{array}$ & $\begin{array}{r}\mathrm{M} \\
33,28 \pm 9,8(21,56-45,0)\end{array}$ \\
\hline Tenis de campo & $\begin{array}{r}\mathrm{M} \\
2,12 \pm 6,3(-9,0-6,5)\end{array}$ & $\begin{array}{r}\mathrm{M} \\
1,98 \pm 0,3(1,65-2,34)\end{array}$ & $\begin{array}{r}\mathrm{M} \\
3,92 \pm 0,2(3,65-4,32)\end{array}$ & $\begin{array}{r}\mathrm{M} \\
8,0 \pm 2,0(5-10)\end{array}$ & $\begin{array}{r}\mathrm{M} \\
28,59 \pm 12,0(9,84-39,14)\end{array}$ \\
\hline
\end{tabular}

$\mathrm{VO}_{2 \text { max: }}$ Consumo máximo de oxígeno

\section{Discusión}

En promedio los estudiantes deportistas de este estudio se encuentran dentro de los límites considerados como normales para colesterol total, LDL y triglicéridos(12), a excepción de los hombres de la selección de porrismo quienes tienen un promedio de $157,1 \mathrm{mg} / \mathrm{dl}$, es decir, con triglicéridos levemente elevados (150$199 \mathrm{mg} / \mathrm{dl})$. En los límites superiores se encontraron algunas personas en el rango de levemente elevado $\mathrm{y}$ elevado, los cuales pertenecían a las selecciones de fútbol, porrismo, tenis de mesa, karate, halterofilia y triatlón. Al respecto Freitas et al.,(16) en un estudio de análisis del perfil lipídico en una población de estudiantes universitarios, encontraron valores elevados de triglicéridos, colesterol total y colesterol asociado a la lipoproteína de baja densidad (LDL-c), los valores reportados de los alumnos fueron $23,0 \%$, $9,7 \%$ y $5,9 \%$, respectivamente.

En relación al HDL, en promedio las mujeres se encuentran en el rango considerado como normal 
(40-60mg/dl) ${ }^{(12) ;}$ sin embargo, en todas las selecciones el límite inferior está por debajo del rango normal. Respecto a los hombres, se observa que en promedio están por debajo del rango los estudiantes deportistas que pertenecen a las selecciones de voleibol, fútbol, porrismo, taekwondo, karate, atletismo y halterofilia y al observar los límites inferiores de las demás selecciones, se puede apreciar que también presentan niveles de HDL que están por debajo de los rangos de normalidad (menor a $40 \mathrm{mg} / \mathrm{dl}$ ). Existe abundante literatura que considera las HDL como un factor protector(17-19) por su rol en el transporte reverso del colesterol además, se describe cómo el ejercicio aumenta esta lipoproteína, sin embargo, estudios como el de García-Cardona et al.,(20) muestran disminución en la concentración de HDL en hombres, y sugieren que para la población joven se deben considerar otros factores que afectan el metabolismo de las HDL como una inadecuada alimentación o un incremento del estrés, entre otros. De igual manera, estudios como el de Ruiz

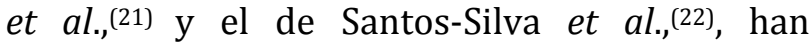
reportado que en sujetos que practican deportes con un alto grado de estrés físico, ocurre un aumento evidente de CT a expensas de las lipoproteínas LDL y una disminución del colesterol HDL-C. Al comparar las concentraciones de HDL de cada selección por sexo se encontraron diferencias estadísticamente significativas $(P \leq 0,05)$ en las selecciones de fútbol, porrismo y tenis de mesa.

Se observó que en promedio los estudiantes deportistas se encuentran dentro de los rangos considerados como normales de índice arterial según el sexo; sin embargo, en algunas selecciones, se presentaron estudiantes con riesgo moderado de sufrir un evento cardiovascular, tales selecciones fueron a nivel femenino fútbol, baloncesto, taekwondo y natación, y a nivel masculino voleibol, futbol, baloncesto, porrismo y tenis de campo.

A nivel de educación superior, los jóvenes que ingresan a los diferentes programas académicos en pregrado, tienen edades que se encuentran entre los 16 y 25 años. Según la reglamentación deportiva de la Asociación Colombiana de
Universidades (Ascun)(23), los estudiantes pueden participar de los juegos zonales y nacionales sólo hasta el límite de los 28 años, lo cual indica que los estudiantes deportistas de la Universidad del Quindío no tendrían inconvenientes de acuerdo a lo encontrado en el estudio, puesto que el promedio de edad es de 21 años, y el promedio mayor fue de 23 años, encontrado en halterofilia. Por lo anterior, los entrenadores en ese caso cuentan con deportistas que podrían estar alrededor de 4 a 5 años disponibles para competir por la Universidad, siendo este el tiempo regular de un estudiante en su carrera y poder proyectarlos de acuerdo a sus condiciones a un proceso de largo plazo, situación que es poco común en este tipo de deportistas, que suelen terminar sus entrenamientos a final de cada semestre, perdiendo la capacidad de una progresión más efectiva de su capacidad, gracias al entrenamiento.

Con respecto a la edad morfológica se encontró que en promedio las mujeres de las selecciones deportivas tienden a presentar una mayor edad con respecto a los hombres (Tabla 2). En términos generales, los hombres muestran mejores promedios a nivel de la edad morfológica con respecto a la cronológica. A nivel masculino, el atletismo y el triatlón muestran los promedios más bajos, ya que su edad cronológica fue de 21 y morfológicamente tienen 19 años en promedio, situación interesante debido a que este tipo de atletas requieren condiciones de fondistas (resistencia aeróbica), lo que nos lleva a analizar la importancia que tiene la actividad practicada (carrera continua) de media y larga duración para la obtención de un buen sistema cardiopulmonar y vascular para mantenerse más joven, tal como lo plantea la OMS(24) al recomendar mínimo media hora de actividad física diaria para mantener una buena salud y evitar enfermedades relacionadas con el sedentarismo.

Con respecto a la talla, en el baloncesto se presentó en promedio una altura de $1,78 \mathrm{~m}$ en los hombres, siendo esta la mayor talla de toda la población, mientras que en voleibol masculino fue de $1,73 \mathrm{~m}$. En el ámbito internacional se encontraron estudios que definen parámetros similares, como el realizado por Gil y Verdoy(4), 
donde se aprecia promedios de $1,76 \mathrm{~m}$ y $1,64 \mathrm{~m}$ en futbol, además $1,89 \mathrm{~m}$ y 1,73m en hombres y mujeres en baloncesto respectivamente, mientras que el voleibol en deportistas juveniles de Brasil tienen promedios de talla de $1,81 \mathrm{~m}^{(25)}$; estos datos que son tomados de universitarios españoles y juveniles brasileños, permiten encontrar diferencias de 9 a $11 \mathrm{~cm}$, evidenciando ser más bajos en Colombia. Cabe resaltar el hecho de que Brasil se considera una potencia suramericana y mundial en todos los deportes y en España se han demostrado avances en los procesos formativos de sus deportistas . Para el caso de las disciplinas deportivas individuales, la talla tuvo un comportamiento dentro del promedio la normal para Colombia con estaturas que oscilaban entre $1,50 \mathrm{~m}$ y $1,73 \mathrm{~m}$.

A nivel universitario se ha encontrado el aumento de problemas de obesidad debido a la inactividad y a los malos hábitos alimenticios, como menciona Lorenzini et al.,(26) los estudiantes universitarios son unos de los sectores poblacionales más educados, no obstante, aparentemente no escapan al problema de la obesidad; para el caso de los deportistas universitarios, el peso no escapa de sus desórdenes debido principalmente a la situación de descontrol alimenticio, que no permite un peso ideal, caso poco aceptable, entendiendo que como deportistas podrían tener mayor control debido al ejercicio. Para el caso de la masa, este análisis debe ir acompañado del IMC, pues permite hacer un análisis más adecuado, aunque no definitivo del cuerpo, y debe ser ratificado el hecho que el sobrepeso o la desnutrición es debido al exceso o falta de grasa corporal(27).

Según el consenso de la Sociedad Española para el Estudio de la Obesidad (SEEDO) el rango para estar saludable o normal está entre 18,5$24,99 \mathrm{~kg} / \mathrm{m}^{2}$, por encima se está en sobrepeso y por debajo en peso insuficiente( ${ }^{(28)}$. Al respecto los deportistas de la Universidad están en promedios normales de IMC, sin embargo, se encontraron datos en baloncesto y tenis de campo femenino de 28,62 y $26,28 \mathrm{~kg} / \mathrm{m}^{2}$ respectivamente, indicando que hay un posible caso de sobrepeso en estos grupos.
Con respecto al ICC brinda la posibilidad de reconocer los riesgos de enfermedades cardiovasculares que existen en las personas. Los parámetros de la $\mathrm{OMS}^{(14)}$ indican que los hombres con 0,95 o menos y las mujeres con 0,8 o menos tienen muy bajo riesgo de sufrir algún episodio cardiaco; mientras que si son mayores las posibilidades son altas. Para el grupo de deportistas el ICC se encuentra en promedios que oscilan entre muy bajos y bajos $(0,78$ hasta 0,91 en hombres y 0,76 hasta 0,84 en mujeres), sin embargo, en la selección de fútbol femenina se encontró un promedio el ICC de 0,87 , entendiendo esto como un alto riesgo de sufrir problemas cardiovasculares, estos casos deben ser analizados de forma más precisa por sus entrenadores.

Según Malagón(27), la cantidad normal de grasa corporal para el hombre debe ser del 15\% y para la mujer del $22 \%$, considerándose obesos los hombres que contienen más del $25 \%$ y las mujeres más del 33\%. En varones jóvenes no atletas la grasa corporal representa alrededor del $17 \%$ del peso corporal; para las mujeres la cifra correspondiente es aproximadamente de un 26\%. Tanto la cantidad como la distribución de la grasa han sido identificadas en varios estudios por su cercana relación con los datos de morbilidad y mortalidad. En el caso de los deportistas de la Universidad del Quindío, se encontró en mujeres que practican baloncesto y tenis de mesa tienen porcentajes de grasa promedio de 30,62 y 32,4\%, lo cual indica de acuerdo a lo planteado por Malagón(27), que estarían en riesgo de llegar a alcanzar niveles de sobrepeso, hecho preocupante, ya que son deportistas; al relacionar este con el porcentaje muscular $(39,57$ y $37,97 \%$ respectivamente) se advierte sobre la necesidad de mejorar el proceso desde el punto de vista de la fuerza, con la cual daría la posibilidad de aumentar masa muscular, quitándole espacio a la masa grasa. En porrismo, se encontró un promedio de $22,73 \%$ de grasa y de musculo de $47,33 \%$, estando dentro los rangos normales; en el caso de los hombres, manejaron porcentajes que oscilaron entre el 20 y el $25 \%$; se puede decir que son promedios normales, sin embargo, los promedios deberían mejorar de acuerdo a los niveles grasos de deportistas de rendimiento, en 
los cuales sus niveles de grasa no sobrepasan el $10 \%$ en hombres y $20 \%$ en mujeres(27).

Algunos deportistas universitarios representan a la región a nivel nacional, pero otros representan la institución sin necesariamente ser de rendimiento, lo que causa que los porcentajes no se manejen en niveles adecuados para deportistas. Los deportes individuales como el triatlón, karate y taekwondo presentan promedios entre 21 y $22 \%$ de grasa y en disciplinas de conjunto el voleibol y el baloncesto con el 22 y $23 \%$. Relacionando esto con el porcentaje muscular, se puede decir que el promedio está sobre el $40 \%$ en los deportes mencionados, planteando la posibilidad de buscar la mejora a nivel muscular de los individuos que están subiendo el promedio de grasa y tratando de llegar a los ideales musculares de un 50\%, que sería lo adecuado para la composición corporal, más aun comparándolos con datos de deportistas nacionales que manejan rangos de grasa corporal máximos del $12 \%$ en hombres y del $19 \%$ en mujeres(27).

En los estudiantes deportistas de la Universidad del Quindío, se encontraron promedios en estos tres componentes en hombres y mujeres que oscilan entre 5 y $6 \%$ en el porcentaje de piel, 9 y $12 \%$ en el porcentaje óseo y 12 y $14 \%$ en el porcentaje residual, planteando una normalidad y dando a entender que son parámetros que se mantienen en los seres humanos, siendo principalmente los que más varían los componentes grasos y muscular. De acuerdo a estudios realizados a universitarios chilenos sobre su composición corporal, nos encontramos en la región dentro de los parámetros lógicos de estos tres componentes ${ }^{(29) .}$

Con respecto a las capacidades condicionales, se sabe que la flexibilidad es una capacidad del aparato locomotor que expresa la amplitud de movimientos, y depende de las propiedades anatómicas y fisiológicas de músculos y articulaciones(30). Esta capacidad es históricamente a la que menos valor se le asigna en el momento de la preparación del entrenamiento de los deportistas; según Platonov y Bulatova(31), un mal nivel de desarrollo de la flexibilidad disminuye los resultados del entrenamiento para desarrollar otras capacidades motoras. Así pues, está demostrado que la eficacia de la preparación de fuerza, aumenta en gran parte cuando aumenta la movilidad. Algunos deportes como la gimnasia, permiten en el trabajo de base utilizar la flexibilidad para el desarrollo óptimo de su deporte; sin embargo, ni en la Universidad del Quindío, ni a nivel general se incluye este en las competencias de Ascun Deportes.

De acuerdo a lo encontrado en la investigación, se pudo determinar que las mujeres de taekwondo, los hombres de porrismo y los de karate, presentaron los mejores resultados a nivel de flexibilidad; tal vez esto, debido a que en el caso del porrismo requieren trabajos continuos de flexibilidad para sus rutinas, mientras que karate y taekwondo para sus combates deben exigir al máximo la extensión de sus extremidades, principalmente las inferiores. Por el contrario, triatlón y mujeres de natación y baloncesto, presentaron los menores resultados en la evaluación, dando a entender, que tal vez, es poco lo que se dedica a la flexibilidad.

Al comparar con estudios internacionales relacionados con la flexibilidad(30), se encontró que los deportistas de la Universidad del Quindío tienen un nivel bajo; por esta razón se invita a incluir dentro de los procesos de planificación, espacios que permitan un trabajo más controlado de la flexibilidad, tal como lo plantea Platonov y Bulatova(31), "el grado de desarrollo de la flexibilidad es uno de los factores más importantes que determinan el nivel del deportista en diferentes modalidades, una movilidad articular insuficiente limita el nivel de los índices de fuerza, velocidad y coordinación; provoca una disminución de la economía y suele ser la causa de lesiones musculares y ligamentosas". Además, la clasificación de Hoeger citado por George et al.,(32) sigue advirtiendo que los deportistas de la Universidad del Quindío están por debajo de los estándares internacionales, corroborando la necesidad de implementar mejores programas para esta capacidad. 
La fuerza es la capacidad condicional definida por algunos autores como la más importante y la que permite que las demás puedan avanzar de manera favorable. La fuerza de los músculos determina en gran medida la rapidez de los movimientos del hombre, desempeña además, un gran papel en las actividades que requieren resistencia $y$ agilidad(33). Estos argumentos permiten analizar los resultados encontrados en el salto realizado por los deportistas de la Universidad del Quindío, en cuanto a la relación constante de la fuerza con las demás capacidades, puesto que los hombres de la selección de karate con un promedio de $2,35 \mathrm{~m}$, fueron los deportistas que presentaron el promedio mayor, esto puede estar asociado al desarrollo de la flexibilidad mencionado. Platonov y Bulatova(31), plantean que "paralelamente al desarrollo de la fuerza, se crean premisas para incrementar el nivel de velocidad, flexibilidad y coordinación". Seguido a este grupo se encontraron los hombres de porrismo $(2,3 \mathrm{~m})$, con valores de flexibilidad también altos, por lo que se podría hacer énfasis en la influencia de ésta en el resultado de la prueba de fuerza, además de los trabajos explícitos de fuerza explosiva que vienen desarrollando, muy utilizado por estas disciplinas deportivas.

Las selecciones masculinas de baloncesto y voleibol, obtuvieron los resultados mayores, concordando de alguna forma con las dinámicas del deporte (fuerza explosiva); sin embargo, se considera la posibilidad de mejorar este aspecto si se tiene en cuenta la flexibilidad como un factor adicional a la fuerza que permita fortalecer esta capacidad condicional. La halterofilia, obtuvo un promedio un poco por debajo de los anteriores, a pesar de que su desempeño requiere de desarrollo de fuerza y está supeditada a la técnica. Según Artiles y Rodríguez(33) durante el proceso de entrenamiento de la halterofilia, se busca desarrollar la técnica racional de los ejercicios competitivos, la fuerza, rapidez, flexibilidad, coordinación, voluntad, entre otras, lo cual invita a tener en cuenta el trabajo de todas las capacidades físicas con el fin de avanzar en su desarrollo, permitiendo alcanzar mejores rendimientos.
Los hombres tienden a desarrollar mayores niveles de fuerza que las mujeres, y esto llevó a que ellas obtuvieran menores resultados en todas las disciplinas deportivas, sin embargo, se debe insistir en la organización adecuada de su trabajo, puesto que pueden alcanzar altos niveles de rendimiento. "Generalmente cuando la mujer es sometida a actividades físicas con una adecuada dosificación, las diferencias morfo-funcionales con respecto al hombre pueden ser reducidas e incluso, pueden presentar índices de desarrollo físico y funcional superiores a hombres no entrenados $o$ con cierto nivel de entrenamiento"(33).

La velocidad se concibe según Hahn citado por Taborda y Nieto(34) como la capacidad del ser humano de realizar acciones motrices con máxima velocidad y dentro de las circunstancias, en un tiempo mínimo. Respecto a la velocidad, los deportistas en los 30 metros obtuvieron tiempos que se consideran en promedios normales. Las disciplinas deportivas requieren por su dinámica competitiva el perfeccionamiento de los diferentes tipos de velocidad (reacción, gestual y desplazamiento), que le permiten mejorar aspectos que van a favor de su rendimiento, por lo cual, la valoración de esta capacidad en la Universidad del Quindío, permite encontrar disciplinas como el futbol (masculino) y tenis de mesa (femenino) entre lo más rápidos, mientras que triatlón con hombres y karate en mujeres los que más tiempo utilizaron en el desplazamiento, lo cual invita a perfeccionar este aspecto importante en la preparación de los deportistas.

Con relación a los datos estandarizados de Davis(35), a excepción de triatlón y halterofilia los grupos masculinos en cada una de las disciplinas deportivas están dentro del rango de excelente, así como las mujeres de tenis de mesa, voleibol, futbol y baloncesto. Mientras que las mujeres de squash, tenis de campo, taekwondo, porrismo y natación, se encontraron en un rango de promedio bajo y medio. Como punto a resaltar, el triatlón, que fue destacado en resistencia, evidencia su especialización en las largas distancias, desentendiéndose un poco de las distancias cortas donde la velocidad toma relevancia. 
La resistencia es la capacidad física y psíquica de soportar el cansancio frente a esfuerzos relativamente largos y/o la capacidad de recuperación rápida después de los esfuerzos(35). Todas las disciplinas deportivas necesitan de una formación en resistencia adecuada que permita al deportista desempeñarse de buena forma, tal como lo plantea Grosser et al.(35), esta capacidad de condición física constituye un requisito decisivo para la consecución de muchos rendimientos deportivos y también para el desarrollo de capacidad de rendimiento deportivo. Es así como la evaluación llevada a cabo por los deportistas de la Universidad del Quindío, se basó en la posibilidad de estimar a través de un test de potencia aeróbica máxima (Test Legger(15)), el consumo máximo de oxígeno y de esa forma perfilarlos en su condición.

Este test evidenció desde el punto de vista de la resistencia distintos comportamientos tanto en mujeres como en hombres y entre los deportes individuales y de conjunto; se resalta que los hombres de voleibol, fútbol, baloncesto, taekwondo, natación, atletismo y triatlón alcanzaron niveles adecuados de $\mathrm{VO}_{2 \max }$, (que se define según Grosser et al(29) como aquella cantidad de oxigeno/min que se inspira como estado equilibrado), mientras que los hombres de halterofilia se mantuvieron bajos. Por el lado de las mujeres, se resalta el voleibol con las deportistas que mejor condición física obtuvieron en contra posición con las tenista de campo. Se puede enfatizar en estos casos la continuidad del entrenamiento como uno de las variables a tener en cuenta a la hora de mejorar la condición física, puesto que la frecuencia semanal en que entrenan, como la posibilidad de sostener los ciclos de entrenamiento de semestre a semestre, no es igual en todas las disciplinas deportivas y esto puede contribuir a la pérdida de la resistencia y de otras capacidades condicionales de manera sistemática, conllevando de esta forma a no mantener un nivel adecuado durante la temporada, situación que debe ser analizada por los entrenadores.

\section{Conclusión}

A pesar de que en promedio el perfil lipídico de los deportistas se encuentra dentro de los rangos considerados como normales, es necesario tener cuidado con el porcentaje adiposo, para ello el entrenamiento deberá estar acompañado de un régimen de dieta saludable, además en los entrenamientos desarrollar ejercicios que permitan aumentar la flexibilidad en todas las disciplinas.

\section{Agradecimientos}

A los deportistas, entrenadores, al Grupo de Investigación en Fisiología de la Actividad Física y la Salud (GIFAS), al Grupo de Investigación en Enfermedades Metabólicas (GECAVYME), a la dirección de Bienestar Institucional de la Universidad del Quindío y a los Licenciados Martha Nidia Cardona y Pedro Luis Campos.

\section{Conflicto de intereses}

Los autores declaran que no existen conflictos de intereses.

\section{Referencias}

1. Zúñiga U, de León Fierro L. Somatotipo en futbolistas semiprofesionales clasificados por su posición de juego. Rev Int Cienc Deporte. 2007;III(9):29-36.

2. García J, Cañadas M, Parejo I. Una revisión sobre la detección y selección del talento en balonmano. ebalonmano.com Rev Digit Deport. 2007;3(3):39-46.

3. Moreno P, Manonelles C. Manual de Cineantropometría. España: NEXUS MEDICA EDITORES SL; 2012. 434 p.

4. Gil J, Verdoy P. Caracterización de deportistas universitarios de fútbol y baloncesto: antropometría y composición corporal. e-balonmano.com Rev Digit Deport. 2010;7(1):39-51.

5. Hagberg J, Park J, Brown M. The role of exercise training in the treatment of hypertension. Sport Med. 2000;30:193-206.

6. Franklin $\mathrm{BA}, \mathrm{Ph} \mathrm{D}$, Cardiology $\mathrm{P}$, Rehabilitation $\mathrm{C}$, Durstine JL, Ph D. Impact of diet and exercise on lipid management in the modern era. Best Pract Res Clin Endocrinol Metab [Internet]. Elsevier Ltd; 2017;28(3):405-21. Available from: http://dx.doi.org/10.1016/j.beem.2014.01.005

7. Caro J, Navarro I, Romero P, Lorente RI, Antonia M, Martínez-hervás S, et al. Efecto metabólico del ejercicio físico regular en la población sana. Endocrinol y Nutr [Internet]. SEEN; 2017;60(4):167-72. Available from: http://dx.doi.org/10.1016/j.endonu.2012.11.004

8. Blazek A, Rutsky J, Osei K, Maiseyeu A, Rajagopalan S. Exercise-mediated changes in high-density lipoprotein : 
Impact on form and function. Am Heart J [Internet]. Mosby, Inc.; 2013;166:392-400. Available from: http://dx.doi.org/10.1016/j.ahj.2013.05.021

9. Berenson G, Srinivasan S, Webber L. Cardiovascular risk in early life: the Bogalusa Heart Study. Current Concepts, a SCOPE publication. Kalamazoo. Michigan: Upjohn Company, Kalamazoo; 1991. 41-53 p.

10. Marfell-Jones $\mathrm{M}$, Olds $\mathrm{T}$, Stewart A, Carter L. International Standards for Anthropometric Assessment. South Africa: ISAK; 2006. 131 p.

11. Ministerio de Protección Social. Presidencia de la República. RESOLUCION NUMERO 8430 DE 1993. Colombia; 1993 p. 1-19.

12. ATP III. ATP III Guidelines At-A-Glance Quick Desk Reference. ESTADOS UNIDOS; 2001.

13. Organización Mundial de la Salud (OMS). Obesidad y sobrepeso. Nota descriptiva $\mathrm{N}^{\circ} 311.2016 ; 1$.

14. WHO expert consultation. Appropriate body-mass index for Asian populations and its implications for policy and intervention strategies. Lancet. 2004;363(9403):15763.

15. Alba A. Test funcionales, cineantropometria y prescripción en el entrenamiento en el deporte y la actividad física. España: Editorial Kinesis; 2012.282 p.

16. Freire de Freitas RWJ, Moura de Aráujo MF, Soares Lima AC, Rodrigues Pereira AM, Coelho Damasceno AM. Study of Lipid profile in a population of university students. Rev Latino-Am Enferm. 2013;21(5):1151-8.

17. Pérez-Mendez O. Lipoproteínas de alta densidad (HDL). ¿Un objetivo terapéutico en la prevención de la aterosclerosis? Arch Cardiol México. 2004;74(1):53-67.

18. Lira FS, Yamashita AS, Uchida MC, Zanchi NE, Gualano B, Martins E. Low and moderate, rather than high intensity strength exercise induces benefit regarding plasma lipid profile. Diabetol Metab Syndr. 2010;2(31):1-6.

19. Ponce Y, Ponce A, Rodríguez A, Llanes C. Las lipoproteínas de alta densidad : protectoras vasculares contra la aterosclerosis. CorSalud. 2013;5(4):366-78.

20. García-Cardona DM, Nieto O, Landázuri P. Efecto del ejercicio sobre las subpoblaciones HDL, la enzima lecitina-colesterol acil-transferasa y la proteína transportadora de ésteres de colesterol en estudiantes de Medicina. Rev Colomb Cardiol [Internet]. Sociedad Colombiana de Cardiología y Cirugía Cardiovascular; 2015;22(6):277-84. Available from: http://dx.doi.org/10.1016/j.rccar.2015.04.007

21. Ruiz JR, Mesa JLM, Mingorance I, Rodríguez-cuartero A, Castillo MJ. Deportes con alto grado de estrés físico afectan negativamente al perfil lipídico plasmático. Rev Esp Cardiol. 2004;57(6):499-506.

22. Santos-Silva A, Rebelo M, Castro E, Belo I, Guerra A, Rego C, et al. Leukocyte activation, erythrocyte damage, lipid profile and oxidative stress imposed by high competition physical exercise in adolescents. Clin Chim Acta. 2001;306(1-2):119-26.

23. Asociación Colombiana de Universidades A. Reglamentos técnicos por disciplina deportiva, juegos universitarios nacionales. Asoc Colomb Universidades. 2012;104.

24. Organización Mundial de la Salud (OMS). Recomendaciones mundiales sobre actividad física para la salud. Suiza: Organización Mundial de la Salud; 2010. $58 \mathrm{p}$.

25. De Araujo B, De Araujo S, Toledo I, Moreira P, Ferreira de Miranda H, Knakfuss M. Antropometria e somatotipo: fatores determinantes na seleção de atletas no voleibol brasileiro. Rev Bras Ciênc Esporte. 2011;333:733-46.

26. Lorenzini R, Betancur-ancona DA, Chel-guerrero LA, Segura-Campos MR, Castellanos-Ruelas AF. Estado nutricional en relación con el estilo de vida de estudiantes universitarios mexicanos. Nutr Hosp. 2015;32(1):94-100.

27. Malagon C. Manual de antropometría. España: Editorial Kinesis; 2004. 165 p.

28. Salas-salvadó J, Rubio MA, Moreno B, Grupo colaborativo SEEDO. Consenso SEEDO 2007 para la evaluación del sobrepeso y la obesidad y el establecimiento de criterios de intervención terapéutica. Med Clin. 2007;128(5):184-96.

29. Rodríguez-Rodríguez F, Santibañez-Miranda $M$, Montupin-Rozas G, Chávez-Ramírez F, Solis-Urra P. Diferencias en la composición corporal y actividad física en estudiantes universitarios según año de ingreso. Univ y Salud. 2016;18(3):474-81.

30. Valbuena García R. Evaluación y normas para la clasificación de la capacidad física "Flexibilidad" considerando personas entre 9 y 50 años de edad pertenecientes al Distrito Capital de la ciudad de Caracas. Rev Invest (Guadalajara). 2007;31(61):121-42.

31. Platonov V, Bulatova M. La preparación física. 4th ed. España: Editorial Paidotribo; 2017. 408 p.

32. George J, Fisher G, Vehrs P. Test y pruebas físicas. 4 th ed. España: Editorial Paidotribo; 2005. 310 p.

33. Artiles H, Rodríguez H. Entrenamiento de la fuerza con sobrecargas. 2nd ed. España: Editorial Kinesis; 2006. $240 \mathrm{p}$.

34. Taborda J, Nieto L. El desarrollo de la velocidad en el niño. Teoría y práctica. España: Editorial Kinesis; 2005. $254 \mathrm{p}$.

35. Gosser M, Bruggemann P, Zintl F. Alto rendimiento deportivo, planificación y desarrollo. España: Ediciones Martínez Roca, S.A; 1989. 223 p. 